\title{
Geospatial analysis of household spread of Ebola virus in a quarantined village - Sierra Leone, 2014
}

\author{
B. L. GLEASON ${ }^{1}$, S. FOSTER ${ }^{2}$, G. E. WILT ${ }^{2}$, B. MILES ${ }^{2}$, B. LEWIS ${ }^{2}$, \\ K. CAUTHEN ${ }^{3}$, M. KING ${ }^{4}$, F. BAYOR ${ }^{5}$, S. CONTEH $^{5}$, T. SESAY $^{5}$,

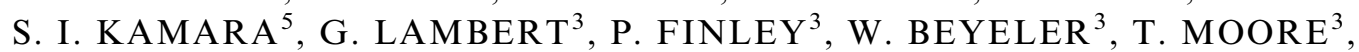 \\ J. GAUDIOSO ${ }^{3}$, P. H. KILMARX ${ }^{1}$ AND J. T. REDD ${ }^{1}$ \\ ${ }^{1}$ Center for Global Health, Centers for Disease Control and Prevention (CDC), Atlanta, Georgia, USA \\ ${ }^{2}$ Agency for Toxic Substances and Disease Registry (ATSDR), Atlanta, Georgia, USA \\ ${ }^{3}$ Sandia National Laboratories (SNL), Albuquerque, New Mexico, USA \\ ${ }^{4}$ Center for Surveillance, Epidemiology and Laboratory Services, CDC, Atlanta, Georgia, USA \\ ${ }^{5}$ Ministry of Health and Sanitation of Sierra Leone (MoHS), Makeni, Sierra Leone
}

Received 8 February 2017; Final revision 14 July 2017; Accepted 31 July 2017;

first published online 22 August 2017

\section{SUMMARY}

We performed a spatial-temporal analysis to assess household risk factors for Ebola virus disease (Ebola) in a remote, severely-affected village. We defined a household as a family's shared living space and a case-household as a household with at least one resident who became a suspect, probable, or confirmed Ebola case from 1 August 2014 to 10 October 2014. We used Geographic Information System (GIS) software to calculate inter-household distances, performed space-time cluster analyses, and developed Generalized Estimating Equations (GEE). Village X consisted of 64 households; $42 \%$ of households became case-households over the observation period. Two significant space-time clusters occurred among households in the village; temporal effects outweighed spatial effects. GEE demonstrated that the odds of becoming a case-household increased by $4.0 \%$ for each additional person per household $(P<0.02)$ and $2.6 \%$ per day $(P<0 \cdot 07)$. An increasing number of persons per household, and to a lesser extent, the passage of time after onset of the outbreak were risk factors for household Ebola acquisition, emphasizing the importance of prompt public health interventions that prioritize the most populated households. Using GIS with GEE can reveal complex spatial-temporal risk factors, which can inform prioritization of response activities in future outbreaks.

Key words: Ebola, geospatial analysis, Generalized Estimating Equations (GEE), Geographic Information System (GIS), epidemiology.

\section{INTRODUCTION}

The West African Ebola virus disease (Ebola) epidemic was unprecedented in complexity and scale

\footnotetext{
* Author for correspondence: B. L. Gleason, 2160 Freetown Place, Dulles, VA 20189, USA (Email: yer7@cdc.gov)
}

[1]. Public health strategies for control of Ebola outbreaks, based on decades of experience, include identifying and isolating cases, monitoring contacts, and breaking chains of transmission [2, 3]. Despite these efforts, the 2014 Ebola epidemic proved difficult to control, and led to over 28000 cases and 11000 deaths [4]. Past studies have focused on individual-level risk factors for Ebola acquisition; however, because 
many public health interventions occur at a community or household level, risk factor analysis at those levels may be informative. Furthermore, since people in a village setting tend to aggregate in social units, examining risk factors for Ebola within a social unit such as a household might reveal new patterns of risk. Determining household risk factors can thereby inform household-level interventions, rather than relying on individual-level data to guide broader public health actions.

Use of quarantine to separate contacts of persons with Ebola to limit Ebola virus transmission was part of the Ebola control strategy in Sierra Leone, in which households and sometimes entire villages were quarantined (online Supplementary Appendix I) [5, 6]. Households were quarantined even if the infectious individual was no longer in the household (e.g. taken to an isolation unit) so that household contacts who had the potential to develop Ebola could be monitored $[6,7]$. Household quarantines were designed to serve as a physical barrier to prevent persons residing outside of the quarantined household from being exposed to Ebola virus.

In Village X of Sierra Leone, a traditional household structure consists of one or more houses aggregated around one main house in which the head of household resides. Since households have spatial relationships to one another that may affect transmission of a pathogen, such as Ebola virus, which is spread by direct person-to-person contact, geospatial analysis can be used to explore complex transmission patterns [8]. In addition, risk of transmission of Ebola virus is not static over time; there is evidence that factors such as illness stage can affect infectivity [9]. Given that infectiousness increases over the course of illness on an individual level, time might also be an important risk factor for Ebola transmission on a household level. We therefore sought to describe geospatial and temporal risk factors for household-to-household spread of Ebola virus in a village that had been quarantined in its entirety by local public health authorities.

In Village X, located in a rural part of Sierra Leone, the earliest symptom onset date for an Ebola case was 10 August 2014, and the first household was quarantined on 12 September 2014. Because of the rapidly rising number of Ebola cases in Village X, a villagewide quarantine was initiated on 18 September 2014 by local health authorities. Other public health response measures that occurred concurrently with the village-wide quarantine in Village $\mathrm{X}$ included community education with dissemination of messages such as
'Ebola is real' and 'no touch,' the 'House-to-House' national campaign ${ }^{1}$ [10], enhanced surveillance, and reintegration of Ebola survivors from treatment centres into the community.

We collected household-level data, including geospatial data, during the Ebola outbreak in Village $\mathrm{X}$ to determine household risk factors for spread of Ebola virus in the context of the active public health interventions being implemented as part of the outbreak response.

\section{METHODS}

We collected household data on all 64 households in Village $\mathrm{X}$ on 8 October and 10 October 2014 to define a retrospective cohort. We defined a household as a family's shared living space associated with a single head of household, which could consist of one or more houses. We defined a case-household as a household in Village $\mathrm{X}$ with at least one resident who became a suspect, probable, or confirmed Ebola case between 1 August 2014 and 10 October 2014, as defined by the Ministry of Health and Sanitation (MoHS) of Sierra Leone and consistent with the World Health Organization (WHO) Ebola case definitions [7]. The day of symptom onset of the earliest case in a case-household defined the day on which we considered a case-household to be incident.

We conducted in-person interviews alongside local public health officials, including one contact tracer who lived in Village $X$, to determine the number of persons per household (as a proxy for household crowding), number of houses per household, number of latrines per household, number of rooms per household, household quarantine status, and other pertinent household characteristics (online Supplementary Appendix II). The name, age, sex, and date of symptom onset for the index case for each case-household were collected during the interviews and confirmed by comparing these data with the Ebola database at the District Health Management Team (DHMT) office, which was maintained using the Viral Hemorrhagic Fever (VHF) application of Epi Info 7·1·4.0 (CDC, Atlanta GA).

We assigned location data to households using handheld GPS units for onsite geocoding, then confirmed the GPS coordinates with aerial photos from Google Earth $^{\mathrm{TM}}$. Using Esri ArcGIS 10.2 Geographic Information System (GIS) software (Environmental

1 The house-to-house campaign was conducted for 3 days in September 2014 as a national intervention to provide Ebola education door-to-door and conduct active case search. 
Systems Research Institute, Redlands, CA), we calculated distances between households as the distance between the household's centers. We performed a Weighted K-Function distance analysis to examine the purely spatial effect of distance on case-household clustering, using point pattern analysis software, PPA1.0c (San Diego State University, CA). Using a Poisson distribution with household as the population and controlling for population size of household as a covariate, we performed Kulldorff's space-time and temporal scans in SaTScan to detect potential casehousehold clusters (online Supplementary Appendix III). The Kulldorf Scan Statistic determines a statistically significant cluster in time and space by a likelihood ratio. This method compares the observed distribution in a study area to a large statistical sample of computer generated random points in the same study area, and identifies a space-time location or time period with a significant excess of cases.

To determine if the distance from a case-household (i.e. potential source-households) affected a household's (i.e. uninfected households susceptible to becoming a case-household) risk of infection, we calculated the daily average distances between casehouseholds and uninfected households over the course of the outbreak from 8 August 2014 to 25 September 2014 in Microsoft Excel (Microsoft Corporation, Redmond, WA) (online Supplementary Appendix IV). We defined potential source-households for a given household as case-households that had symptom onset at least 2 days prior to symptom onset in that given household.

The daily average distance metric is a composite of the distances calculated for each of the 64 households in Village $\mathrm{X}$, and represents the average distance from a given household to all potential source-households on a given day. We then developed distance metrics that included consideration of household quarantine to see if household quarantines modified risk of household Ebola virus acquisition posed by distance to casehouseholds. The average quarantine distance metric was based on the assumption that institution of household quarantine reduces to zero the risk posed by an infected, quarantined case-household. Therefore, for the average quarantine distance metric, we treated quarantined case-households as if they were uninfected and consequently not potential source-households beginning on the day the household quarantine was initiated.

Because the timeliness of instituting household quarantines varied over the course of the outbreak in Village $\mathrm{X}$, we then developed a similar average prompt quarantine distance metric based on the assumption that only household quarantines instituted less than $48 \mathrm{~h}$ after symptom onset of a case-household were effective. The average prompt quarantine distance metric was developed to assess if quarantines instituted within $48 \mathrm{~h}$ were effective in reducing household Ebola virus transmission, given that quarantines instituted greater than $48 \mathrm{~h}$ after symptom onset might have limited effect.

To evaluate the risk of household Ebola acquisition posed by potential risk factors, we performed a generalized estimating equation (GEE) in R3.2 (R Core Development Team, 2008), which focuses on calculating population averaged effects from a correlated or repeated measures dataset. As the time series data from each household were not independent, modeling the data with a GEE was more appropriate than a logistic Generalized Linear Model (GLM). An exchangeable correlation matrix was used with the GEE. Analyses using both average distance and average prompt quarantine distance metrics were conducted (online Supplementary Appendix V). We considered two-sided $P$-values $<0 \cdot 05$ to be statistically significant.

\section{RESULTS}

Village $X$ is approximately $0.7 \mathrm{~km}$ long and $0.4 \mathrm{~km}$ wide, and the population at the beginning of the observation period on 1 August 2014 was 863 persons living in 64 households (97 houses). Median household size was 10 residents (IQR 6-18); however, two households were notably larger than the others with 64 and 55 residents. All 64 households in Village X were geocoded and assessed for household characteristics (Fig. 1). A total of 27 households became casehouseholds ( $42 \%$ cumulative household attack rate) as depicted in the epidemic curve by date of symptom onset for the index case in each case-household (Fig. 2). Over the course of the study period, a median of three persons per case-household (mean of 4.8 persons per case-household) developed Ebola (median intra-household attack rate of 30\%); in the two larger households 30 and 20 persons developed Ebola (attack rates of $46 \%$ and $36 \%$, respectively).

In total 22 of 27 (81\%) case-households were quarantined; the most common reason for case-households not to be quarantined was because the household was vacant after all residents had died or left. The last household quarantine was initiated on 1 October 2014, and the village-wide quarantine was 


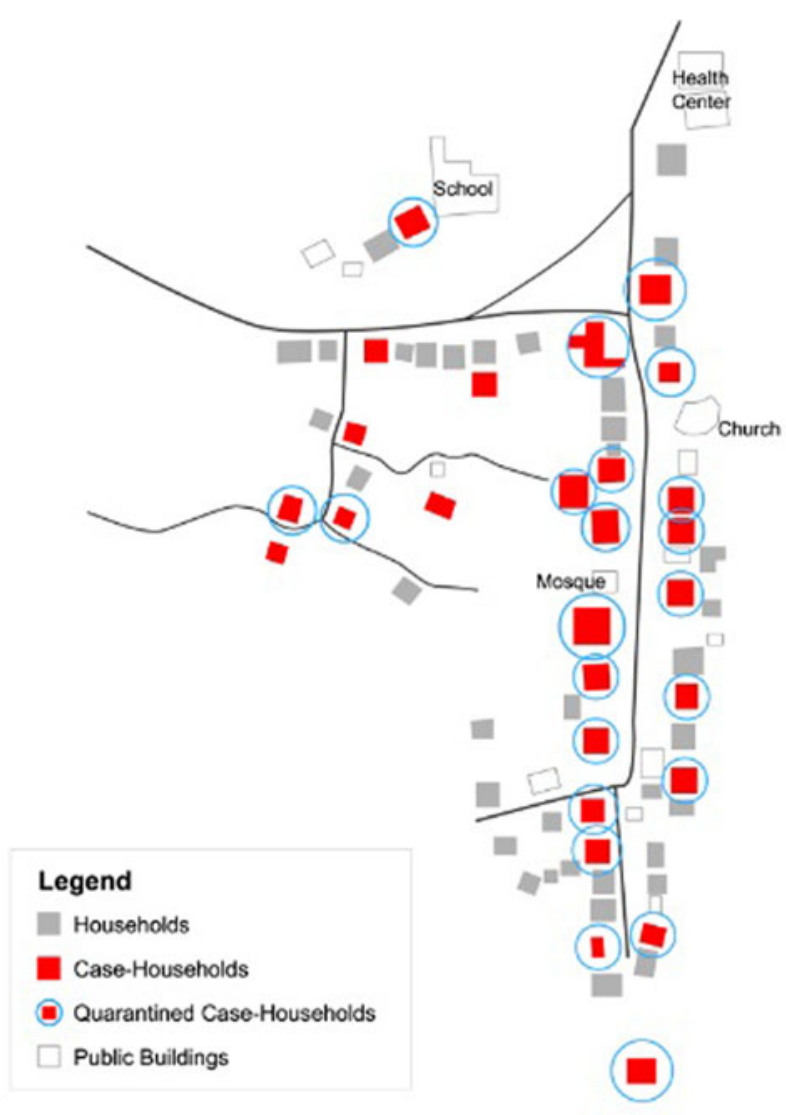

Fig. 1. Schematic map of Village $X$, Sierra Leone, indicating cumulative Ebola case-household status and quarantine status from 1 August 2014 to 10 October 2014. discontinued on 18 October 2014, one month after it was instituted. An animated timeline of household infection and implementation of household and village-wide quarantines in Village $\mathrm{X}$ is depicted in online Supplementary Figure S1.

The mean duration from symptom onset to initiation of household quarantine during the observation period was 10 days (IQR, 2-17 days). However, this measure decreased over the course of the village outbreak, as public health interventions intensified: for case-households in which the index case for a given household had symptom onset prior to 6 September 2014 (i.e. the first half of the outbreak), the mean duration until implementation of quarantine was 15 days; for case-households with symptom onset on or after 6 September 2014, the mean duration until quarantine was 5 days.

\section{Spatial-temporal analysis}

Space-time cluster analysis revealed two statistically significant clusters. A primary cluster contained five infected households out of 10 total households in a $58 \mathrm{~m}$ radius over a 2-day period from Days 32 to 33 (10-11 September 2014; $P=0.013$ ). A secondary cluster contained five case-households out of 25 total households in an $86 \mathrm{~m}$ radius over a 1-day period on Day 27 (5 September 2014; $P=0 \cdot 047$ ). A purely

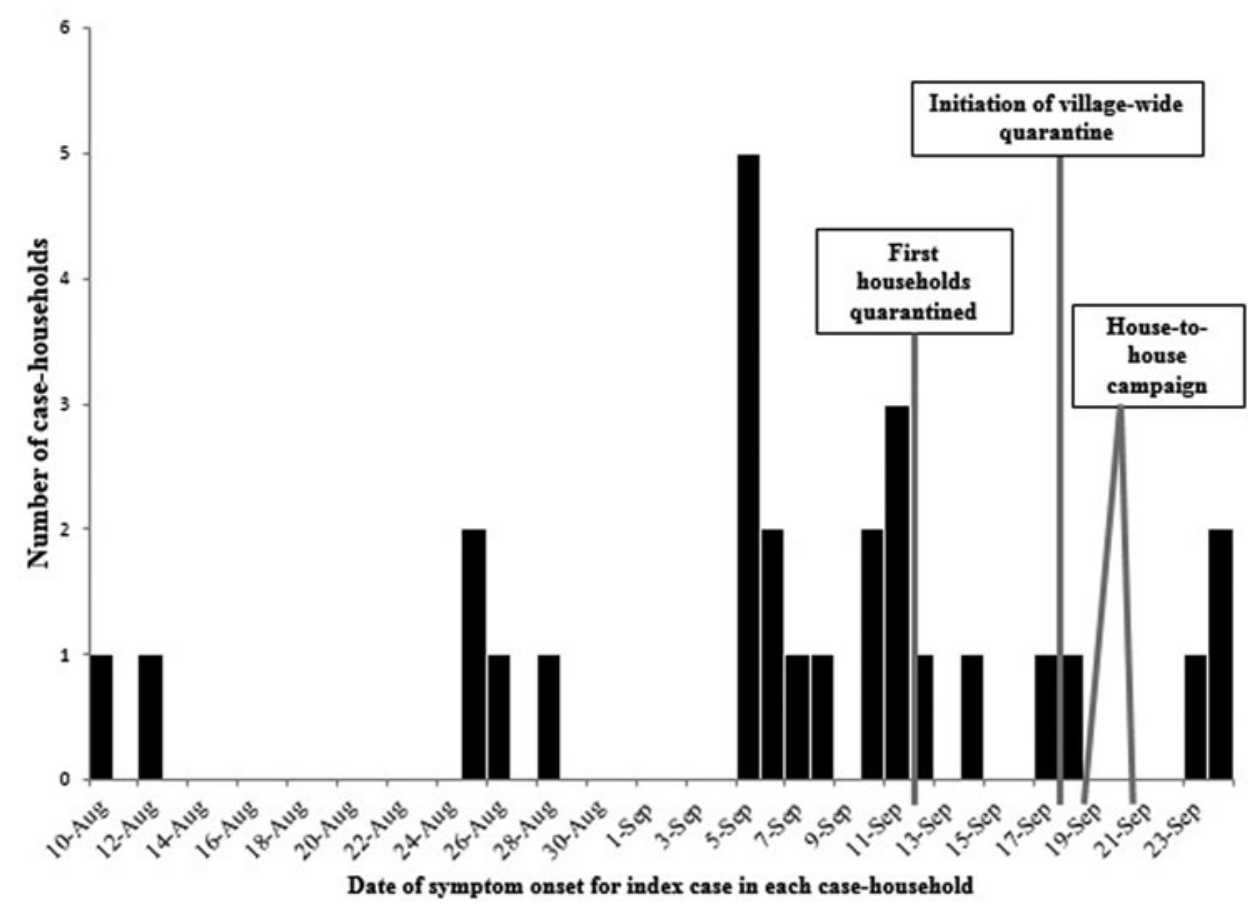

Fig. 2. Case-households $(N=27)$ over time for Village $\mathrm{X}$, Sierra Leone, by week of symptom onset for the index Ebola case in each case-household from August to September 2014. 
temporal cluster analysis revealed one statistically significant cluster, including 15 case-households over an 8-day period from Days 27 to $34(P=0.003)$. Spatial distribution, assessed by the purely spatial Weighted K-Function analysis found no statistically significant spatial clustering of case-households (Fig. 3).

\section{Generalized estimating equations}

Table 1 shows the results of the GEE $(n=865 ; 27$ cases and 838 non-cases) presenting adjusted odds ratios. The GEE demonstrates that when controlling for time and the average distance metric, the odds of a household becoming a case-household increased by $4.0 \%$ for every one person increase in household size $(P<0.02)$. The odds of a household becoming a casehousehold, when controlling for distance and number of persons per household, increased by $2.6 \%$ per day $(P=0 \cdot 06)$. Replacing the average distance metric with the average prompt quarantine distance metric did not modify the odds of household infection. Adding quarantine as an exposure variable did not result in changes to other covariates in any model (data not shown).

\section{DISCUSSION}

Our analysis showed that a greater number of persons per household, and to a lesser extent, passing time were risk factors for household-level Ebola virus infection in Village X. Our spatial, temporal and spacetime cluster analyses demonstrated that the observed space-time clusters were subsets of the more statistically significant temporal cluster and not due to the spatial relationships between the households. Using the GEE, we demonstrated that the risk of infection increased over time during the first 27 days of the outbreak, and the risk of infection was not affected by distance to case-households. Therefore, we conclude that the average distance between households in Village X did not affect the risk of household Ebola acquisition, but the number of persons per household and increasing time did increase risk. These conclusions may inform Ebola response planning because they suggest prioritizing case finding and investigation in households with a greater number of persons, and that outbreak control be performed as rapidly as possible. In resource-limited settings such as the setting of this outbreak, such prioritization strategies may be of particular import. Our animation of this village's

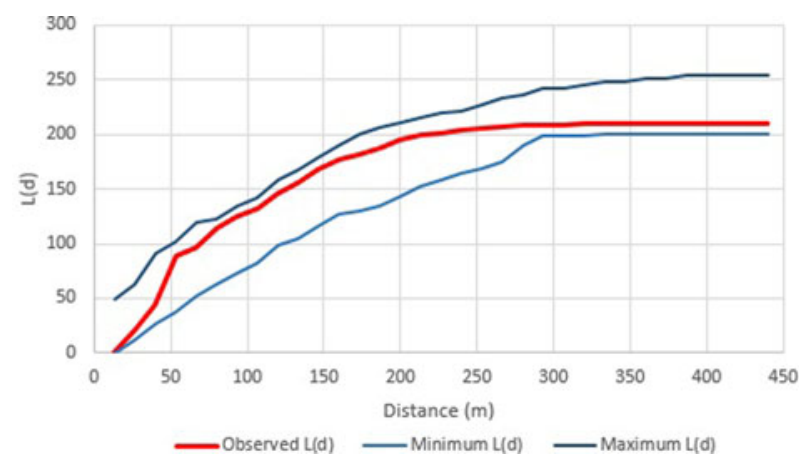

Fig. 3. Spatial distribution of case-households in Village $\mathrm{X}$, Sierra Leone, August to October, 2014. Figure 3 illustrates the random distribution of Ebola case-households as the locations fall between the $95 \%$ confidence limits suggesting neither spatial clustering nor spatial uniformity is occurring (online Supplementary Appendix III). Spatial distribution of case-households was assessed by the Weighted-K function analysis. This function was used to test the hypothesis that the pattern of Ebola case-households among all households in the village is more clustered than chance would have it. We reject our null hypothesis as the observed results fall within the confidence envelope for the weighted $\mathrm{K}$ function results. *Distance is the distance in metres to a neighboring household. ${ }^{* *}$ The $y$-axis depicts the spatial distribution of Ebola case-households in relation to complete spatial randomness, this is signified by the function $L(d)$.

Ebola outbreak (online Supplementary Fig. S1) allows for visualization of household transmission patterns across time and space with detail that cannot be conveyed in a static image or graph.

While the findings that a greater number of persons per household and the passage of time increase risk of household Ebola infection may be intuitive, our study is unique in yielding village-level evidence to substantiate these risks using field data collected on-site in the midst of an Ebola outbreak. The measure of persons per household serves as a proxy for household crowding, but it could also reflect greater possibilities for person-to-person transmission due to mixing among a larger number of people.

This study demonstrates the feasibility of collecting household-level location data in addition to traditional risk factor data during an ongoing outbreak occurring in a remote location with limited resources. The real-time influence of temporality of transmission, as demonstrated by the GEE results, could not be evaluated with logistic regression. Use of GIS data and GEE allowed us to explore the changing spatial relationships between households over time, as households changed from uninfected households to casehouseholds in a dynamic fashion. 
Table 1. Generalized estimating equations for Ebola virus spread at household level in Village X, Sierra Leone, August to October 2014, $n=865$ (27 cases and 838 non-cases)

\begin{tabular}{lllllll}
\hline \hline Variables & OR & Wald test & S.E. & $P$-value & Correlation Est $^{\mathrm{a}}$ & Correlation S.E. $^{\mathrm{a}}$ \\
\hline Multivariate non quarantine GEE & & & & & & \\
$\quad$ Time (outbreak day) & 1.03 & 3.48 & 0.014 & 0.062 & 0.0027 & 0.0063 \\
$\quad \begin{array}{l}\text { Average distance (10 m intervals) } \\
\quad \text { No. of residents }\end{array}$ & 0.978 & 0.31 & 0.004 & 0.577 & & \\
$\quad 1.04$ & 5.59 & 0.017 & 0.018 & & \\
Multivariate prompt quarantine GEE & & & & & & \\
$\quad$ Time & 1.03 & 3.47 & 0.014 & 0.062 & 0.0027 & \\
$\quad$ Average prompt quarantine distance & 0.977 & 0.34 & 0.039 & 0.558 & & \\
$\quad$ No. of residents & 1.04 & 5.50 & 0.017 & 0.01 & & \\
\hline \hline
\end{tabular}

OR, household average odds ratio (population average for the odds of a household becoming a case-household).

${ }^{a}$ The Correlation Estimate and Correlation Standard Error show the estimation and s.E. of the within clustered values. The low values illustrate that the unit of clustering (the household) were not very different from each other.

${ }^{\mathrm{b}}$ Distance is average distance between the household in question and all Ebola case-households that had symptoms at least 2 days prior.

${ }^{\mathrm{c}}$ Quarantine Distance is average distance between the household X and all Ebola case-households that had symptoms at least 2 days prior and were not quarantined prior to the onset date of symptoms in household X.

We suggest that routine geocoding, especially to the household level, be part of standard investigations of future Ebola outbreaks. Geocoding allows for field personnel to locate homes in remote locations that lack standard addresses and helps coordinate disease control efforts conducted by multiple organizations, such as food delivery, provision of water to quarantined homes, and daily monitoring of Ebola contacts. While distance was presumed to be a risk for household transmission, our use of geocoding and GEE demonstrated that, on average, distance was not an important household risk factor for Ebola in Village $\mathrm{X}$. While distance might increase risk in other outbreaks, use of geospatial analyses can evaluate the effect of distance and of public health interventions based on distance.

Our use of GIS and GEE quantified the risks associated with time and persons per household during an Ebola outbreak, supporting the importance of rapid and targeted interventions to control Ebola. Specifically, households with a greater number of persons compared with other households in the village should be prioritized for case finding and isolation based on our findings that these households are at a higher risk of infection. These findings are consistent with the growing body of scientific literature on the 2014 Ebola epidemic demonstrating the necessity of rapid detection, isolation, and treatment of Ebola patients [2, 3, 11]. Other mechanisms needed to achieve Ebola outbreak control and prevent recurrence include establishing incident management systems and emergency operation centres, improving in-country disease surveillance, ensuring rigorous contact tracing, providing capacity for laboratory testing, implementing infection control among healthcare workers, and ensuring a robust global response when a country is overwhelmed by an epidemic [11].

\section{The role of quarantines}

We were not able to reach definitive conclusions on the effect of household quarantines in Village X. Our finding that the mean duration of time from symptom onset until initiation of quarantine decreased from 15 days to 5 days during the observation period suggests an active process of improving the implementation of quarantines during the outbreak. The outbreak in Village X occurred early in the course of the Ebola epidemic, and there were no guidelines on implementation of quarantine in international Ebola preparedness and control documents [3, 12]. In this district, quarantine was typically not enforced until the symptomatic individual had a confirmatory laboratory result for Ebola. At this phase of the outbreak, without a laboratory in the district, it took a mean of 7 days after symptom onset before the result was available [13]. We do not know how effective household quarantines may have been if implemented immediately after the first cases in this village were confirmed.

Additional GEE analyses that included household quarantine found that quarantine, even when implemented promptly, did not modify the risk of household infection. However, because of our assumption that quarantine aims to mitigate the risk posed by 
distance, combined with our finding that distance itself was not an important risk factor for household infection, we were unable to draw further conclusions on the effect of quarantines.

In past Ebola outbreaks, quarantine has not been a key component of infection control measures because it is difficult to implement perfectly and humanely, meaning that individuals may be left without access to basic needs, which may lead to mistrust of public health officials $[5,14]$. This epidemic, however, was unprecedented, and initial projections predicted an exponential rise in cases, so response measures, such as quarantine, differed from prior outbreaks [15-17]. Various forms of quarantine were used in West Africa during this epidemic including household quarantine, village quarantine, and placement of exposed persons in dedicated quarantine facilities separate from residential areas $[5,6,18]$. When Ebola spread to Nigeria in July 2014, for example, health officials utilized an observation unit to house five high-risk Ebola contacts for the duration of their 21-day incubation period [18]. Some of these contacts had crowded home environments, so the group quarantine was deemed to pose a lower risk of Ebola transmission than household quarantine. Nigeria had the resources to implement this intervention with specific protocols to protect those affected; however, authorities acknowledged that 'group quarantine of contacts in a central location might not be workable on a large scale' [18]. Further studies are warranted to develop an evidence base on the effect of quarantines during the 2014 Ebola epidemic.

\section{Limitations}

Our study had several limitations. First, our study by design evaluated household-level rather than individuallevel Ebola virus transmission patterns, which limited our ability to incorporate individual-level risks. Because distance between households is not a proxy for distance between individuals, our findings can only address the relevance of household-level, not personlevel, spatial characteristics. This design was appropriate because the field investigation occurred during an overwhelming outbreak with limited local resources, so accurate, complete individual-level person-time data were unavailable for this village. Without complete individual-level details, however, we could not know when infectious individuals left each casehousehold (e.g. for admission to a healthcare facility or because of death). Thus, we assumed in our models that once a household became a case-household, it remained a potential source of infection to uninfected households for the remainder of the observation period. We made this assumption in consideration of the mean of 4.8 Ebola cases per case-household, and local reports of extended intra-household transmission chains. However, it is possible that some Village $\mathrm{X}$ case-households identified earlier in the course of the outbreak completed their infectious period before the end of the study period.

Second, we were unable to evaluate the influence of unmeasured complex social networks or events that may have affected household risk of infection. Given that Ebola transmission can occur through multiple routes (e.g. point source exposure from a healthcare setting or funeral attendance, exposure from interactions with residents of nearby case-households, or exposure by interactions with residents of distant casehouseholds) it is not possible to differentiate the risk of exposure from each potential route. Third, not having a comparison village limited our evaluation of the effectiveness of specific interventions, such as household quarantine. Fourth, because our GEE evaluates the risk using a distance metric calculated as an average measure for each household over the course of the outbreak, our odds ratio for the effect of time assumes a constant effect throughout the outbreak period, rather than increasing during the initial portion of the outbreak and then decreasing. Assumption of constant effect was necessary in order to build our models, and although the magnitude of the effect of time changed, it was always a risk factor with an effect estimate of greater than one. Finally, our small sample size may have limited the ability to fully assess potential risk factors.

\section{Public health implications}

This study depicted how integrating the assessment of traditional risk factors with geospatial analysis can improve our understanding of the complex dynamics of transmission of a pathogen such as Ebola virus. Our findings provide village-level evidence that time and a greater number of persons per household increased the risk of household acquisition of Ebola virus, highlighting the importance of rapid case finding, accurate case investigation, and with detailed contact identification so that potentially infectious persons can be isolated from other members of the household. Because the two households in Village X that had greater than five times more residents than 
the other households in the village also had higher intra-household attack rates, this suggests that prioritizing larger households during case finding might be an effective strategy.

Our finding that distance was not an important risk factor for household infection suggests that household location is not an effective proxy for information gathered by thorough, in-person contact investigations. Further, while our findings cannot precisely describe the magnitude of the effect of household quarantine if an effect is indeed present, they reinforce strongly the importance of prompt, accurate contact monitoring during the incubation period of Ebola, so that symptoms in contacts can be identified as early as possible and affected persons moved quickly to testing and treatment. Our conclusions are consistent with recent literature, including the strategy of rapid isolation and treatment of Ebola (RITE) in Liberia, which emphasizes the necessity of quickly reaching patients in remote areas. RITE was credited for reducing the time to control outbreaks by half and doubling Ebola survival rates [11].

The principle of least infringement, which offers that 'the least restrictive measures possible should be taken to protect the public's health' can serve as a reminder of the necessity to evaluate the efficacy of quarantines [19]. The task of balancing the protection of population health and maintaining individual liberties is not a new dilemma, but the severity and complexity of the 2014 Ebola epidemic bring attention to this challenging topic $[15,20]$.

Finally, GIS techniques can provide a mechanism to incorporate spatial and temporal risk factors in future outbreak investigations. Field use of GIS technology to the benefit of public health is feasible even in remote settings with limited resources in an ongoing outbreak. Also, as seen in the online animation (online Supplementary Fig. SI), the detailed, household-to-household spread of Ebola virus in this village was much more complex than the village epidemic curve would indicate. Therefore, use of GEE and GIS techniques in tandem with traditional risk factor assessment offers a sophisticated method to visualize and analyse complex household-level details of transmission that can guide public health actions and inform allocation of scarce resources.

\section{SUPPLEMENTARY MATERIAL}

The supplementary material for this article can be found at https://doi.org/10.1017/S0950268817001856

\section{ACKNOWLEDGEMENTS}

The authors thank the following persons who assisted with this study: Sara Snell (GIS Analyst with Geospatial Research, Analysis and Services Program of ATSDR) and John Bangura (MoHS contact tracer for the affected community).This research received no specific grant from any funding agency, commercial or not-for-profit sectors. All work was performed as part of the authors' duties at the Centers for Disease Control and Prevention, Agency for Toxic Substances and Disease Registry, and Sandia National Laboratories. It required no outside funding source.

\section{DECLARATION OF INTEREST}

None.

\section{ETHICAL STANDARDS}

The activities described in this article did not meet the United States federal definition of human subjects research (HSR), as determined by the CDC HSR reviewing staff.

\section{DISCLAIMER}

The findings and conclusions in this report are those of the authors and do not necessarily represent the official position of the Centers for Disease Control and Prevention.

\section{REFERENCES}

1. World Health Organization. WHO advisory group on the Ebola virus disease response: terms of reference. Global Alert and Response. November 2014 (http:// www.who.int/csr/disease/ebola/advisory-groups/adv-groupmeeting2.pdf?ua=1). Accessed 3 June 2017.

2. Dixon MG, Schafer IJ. Ebola viral disease outbreak West Africa, 2014. Morbidity and Mortality Weekly Report 2014; 63: 548-551.

3. World Health Organization. Ebola response roadmap. WHO/EVD/Roadmap/14.1. 28 August 2014 (http://apps. who.int/iris/bitstream/10665/131596/1/EbolaResponse Roadmap.pdf). Accessed 3 June 2017.

4. Centers for Disease Control and Prevention. 2014 Ebola outbreak in West Africa - case counts (http://www.cdc. gov/vhf/ebola/outbreaks/2014-west-africa/case-counts. html). Accessed 3 June 2017.

5. The Assessment Capacities Project. Ebola outbreak in West Africa. Lessons learned from quarantine - Sierra Leone and Liberia. 19 March 2015 (https://www.humanitarianresponse.info/system/files/documents/files/t-acaps_ 
thematic_note_ebola_west_africa_quarantine_sierra_leone_ liberia_19_march_2015.pdf). Accessed 3 June 2017.

6. National Ebola Response Centre. Sierra Leone emergency management program standard operating procedure for management of quarantine. Approved by National Ebola Response Centre Leadership on October 2014, Version 1 (http://www.nerc.sl/sites/ default/files/docs/GOSL\%20QUARANTINE\%20SOP \%20final\%2028_10-14.pdf). Accessed 3 June 2017.

7. World Health Organization. Implementation and management of contact tracing for Ebola virus disease (http://www.who.int/csr/resources/publications/ebola/ contact-tracing/en/). Accessed 10 August 2015.

8. Centers for Disease Control and Prevention. GIS Resources (https://www.cdc.gov/gis/resources.htm). Accessed 3 June 2017.

9. Osterholm MT, et al. Transmission of Ebola viruses: what we know and what we do not know. mBio 2015; 6: e00137-15.

10. Nossiter A. Lockdown begins in Sierra Leone to battle Ebola. The New York Times 2014; 19 September (http://www.nytimes.com/2014/09/20/world/africa/ebolaoutbreak.html). Accessed 3 June 2017.

11. Frieden TR, Damon IK. Ebola in West Africa - CDC's role in epidemic detection, control, and prevention. Emerging Infectious Diseases 2015; 21: 1897-1905. (http://dx.doi.org/10.3201/eid2111.150949). Accessed 3 June 2017.

12. World Health Organization. Ebola virus disease consolidated preparedness checklist, revision 1. 15 January 2015. WHO/EVD/Preparedness/14 Rev.1 (http://apps.who.int/ iris/bitstream/10665/137096/1/WHO_EVD_Preparedness_ 14_eng.pdf?ua=1\&ua=1). Accessed 3 June 2017.
13. Gleason B, et al. Establishment of an Ebola treatment unit and laboratory - Bombali district, Sierra Leone, July 2014-January 2015. Morbidity and Mortality Weekly Report 2015; 64: 1108-1111.

14. Tognotti E. Lessons from the history of quarantine, from plague to influenza A. Emerging Infectious Diseases 2013; 19: 254-259. (http://wwwnc.cdc.gov/eid/ article/19/2/12-0312_article). Accessed 3 June 2017.

15. World Health Organization. Ebola in West Africa: heading for catastrophe? Strong control measures needed to stop steep climb in cases: Ebola at 6 months (http://www.who.int/csr/disease/ebola/ebola-6-months/ west-africa/en/). Accessed 3 June 2017.

16. World Health Organization. Ebola virus disease. Fact sheet No 103. September 2014. (https://web.archive.org/ web/20141222153405/http://www.who.int/mediacentre/ factsheets/fs103/en/). Accessed 3 June 2017.

17. Meltzer MI, et al. Estimating the future number of cases in the Ebola epidemic - Liberia and Sierra Leone, 2014 2015. Morbidity and Mortality Weekly Report 2014; 63: $1-14$.

18. Grigg C, et al. Use of group quarantine in Ebola control - Nigeria, 2014. Morbidity and Mortality Weekly Report 2015; 64: 124.

19. Presidential Commission for the Study of Bioethical Issues. Ethics and Ebola: public health planning and response. Liberty-restricting public health interventions and the principle of least infringement. Washington, D.C.: February 2015, pp. 7 (http://bioethics.gov/sites/ default/files/Ethics-and-Ebola_PCSBI_508.pdf).

Accessed 15 January 2016.

20. Gostin LO, Lucey D, Phelan A. The Ebola epidemic: a global health emergency. JAMA 2014; 312: 1095-1096. 